\title{
A Novel "Monte Carlo" Simulator Aimed to Model High Energy Up-to-date Radiation Sources and Radiation Transport inside Matter
}

\author{
G. Fazio, M. Giaconi and S. Vetruccio \\ Electronic Engineering Department, Faculty of Engineering, University of Rome “Tor Vergata”, Rome 00133, Italy
}

\begin{abstract}
We have faced the challenge of developing a tool devoted to simulate the transport of photons and accelerated particles (ionizing radiation entities) inside matter, where the choice of transporting medium shall be free for the user and non-homogeneities shall be modeled and managed. The solution has been a project that exploits fully statistical Monte Carlo approach, employing a digitalizing board to produce random samples. The system heredities many concepts from electronics devices simulators, but it finds different applications and we present one of them for effectiveness demonstration.
\end{abstract}

Key words: Monte Carlo, automatic simulator, radiation transport, radiotherapy, dose-depth analysis.

\section{Introduction}

We have created a Monte Carlo (MC) simulator prototype; a project aimed to describe the transport of photons and accelerated particles (ionizing radiation entities) inside matter. MC approach is the best one when we face problems with many variables and complexity. This system has given life to an algorithm, useful to compute the evolution of high energy particles in their space of phases as a modality of solution of the Boltzmann's equation of balance.

\section{Materials and Methods}

\subsection{Basics on the Model}

The system is HW-SW implemented using commercial National Instruments tools and devices.

The software has been entirely developed in LabVIEW $^{\mathrm{TM}}$ programming environment, employing also Data acquisition board, managed by a Xilinx ${ }^{\mathrm{TM}}$ FPGA module for sampling of random noise.

The theoretical framework is built up on the

Corresponding author: S. Vetruccio, Ph.D., electronic engineer, research fields: developing of software for simulations of physical models and in railway industry. definition of the space of phases of the particles involved into simulation, i.e. photons, electrons and positrons; all intended as point entities according to the celebrated semi-classical approximation model for transport. $\beta \pm$ radiations are fully characterized by their position in a 6-dimensions space: 3 Dimensions (3D) related to actual position, two angles (spherical coordinates for aiming), and kinetic energy. X-ray or Gammas ray $(\gamma)$ photons have got their state characterized as previously, plus a 3D vector for linear polarization.

The choice of transporting medium is free for the user, so it cannot trivially be modeled as homogeneous. For the proposed solution space must be divided into elementary 3D cells, into which properties can be considered constant with acceptable level of approximation. The smallest these vowels are, the best accuracy is obtainable from the results. Obviously actual cell dimensions depend on hardware resources of the available computing machine.

\subsection{Data Input}

An interaction is an event that can modify the particle's state. The properties of a 3D basic cell are 


\section{A Novel "Monte Carlo" Simulator Aimed to Model High Energy Up-to-date Radiation Sources and Radiation Transport inside Matter}

rigorously related to the probability of interaction, and that interaction is a function of mass attenuation coefficients and with the density of nuclei and orbital electrons in a unit of volume. Our system receives this info from freely available databases ([1]), and from “.jpg” files which contain multiple 2D scans of the target.

Although the system is designed to draw a 3D model of the domain starting from easily available and common X-ray scans. Best approximations would be achievable if we employed very low Energy X-ray for scanning: vacuum tubes with Mo-target are uncommon but could represent a good mean to investigate matter's density with $15 \mathrm{keV}$ peaked photons (in fact calculations for interaction probability are conducted in the $10 \mathrm{keV}$ case, minimum of energy which takes part to our simulations).

The particles' transport phenomena descend from this model and from a collection of parameters taken from validated NIST database: in Ref. [1] we have acquired values of mass attenuation coefficients as a function of medium typology and of various particle energies. The available database let us manage quanta of radiation from $10 \mathrm{keV}$ to $50 \mathrm{MeV}$.

The statistical distributions that describe the initial state of particles are essential input too. The statistical distributions depend on the characterization of the source of radiations and must be imported from technical literature. Statistical distributions can define the initial state of the particle ensemble, enriching the model with information about the energy spectrum and the radiation diagram of the considered source for a given simulation. The software accepts the initial declaration of a 3D point in order to consider it as the center of the target: an automatic software routine makes the pointing assigning to each particle its targeting.

\subsection{Body of the Simulation Software}

Each particle is followed during its journey into the space of phases: from its staring point within such a space, throughout interaction events. According to the radiation transport model, an interaction event can lead to:

- the total disappearance of a particle, event that causes the simulation to go ahead;

- the generation of new particles, put in queue because their history needs to be studied as well;

- the transferring of a certain amount of energy to the traveled path of elementary cells.

Specific quantities will be the results of simulations. The current SW settings look at: the total amount of energy inside the volume of matter, the $\beta \pm$ and $\gamma$ particles exiting from boundaries, obtained after interaction of the primary beam with $3 \mathrm{D}$ domain. These quantities are not time related; so random extractions, part of MC procedures, are continuously devoted to assign a "length of flight (free path)" to each particle.

This time independence, together with the acceptable independence of a particle motion (consider that it is almost impossible that two travelling particles interact each other instead of with the crossed medium), let us not mark the evolution of the "Ensemble" in time domain. So, calculations related to each particle can be independently carried on by our program.

The implemented algorithm tries to simplify the management of the dependency of event probability from particles' energy, choosing a fully statistical approach, and so introducing self-scattering [2] as a possible interaction mode in addition to the main ones.

For $\beta \pm$ particles we have considered the following main modes: multiple Coulombian scattering, bremmstrahlung radiation, annihilation. For photons: Photoelectric effect, Compton, Thompson Scattering, pairs production. We have not added any other phenomena: their probability of occurrence would be so low, that no sensible additional error would be made.

The state of a particle element in the space of phases must be recalculated and updated every time an 
interaction occurs. The mathematical relations that let us compute new states are integral part of the model defined for the simulator: they are deduced from available literature (e.g. Refs. [3, 4]).

\subsection{Hardware Components Part of Simulator}

The history of a single particle, i.e. length of flight, type of interaction, determination of new state, etc... dramatically depends on the range in which a uniformly distributed in $(0,1)$ random number falls. MC simulation is carried out employing an appropriate random float number generator which results to be the core of the system. LabVIEW $^{\mathrm{TM}}$ provides a tool implementing multiplicative congruential algorithm [5], with a pseudo-random list that recycles every $6.75 \times 10^{12}$ extractions. The generator routine is invoked throughout the simulation a lot of times, not much less then the recycling limit. For this reason we have added real time hardware to the project.

We employed an analogical input channel, assigning to a FPGA the task of sampling and quantizing the white band voltage signal generated by thermal noise, outputted from a $100 \mathrm{k} \Omega$ commercial resistor. We ended up obtaining a physical source of completely random float numbers on an embedded module, as a distinguishing feature of our system.

\subsection{Proof of Correct Functionality and Application for the Simulator}

It is imperative to show the reliability of the model and of the built software. We have produced a proof of application in order to validate model and code.

The proposal is referring to validated data, possibly assessed with experimental measures, addressed to refer the amount of released energies inside water-like dense medium from a single radiation beam. In fact, the released energy is a function of the transport phenomena happened to photons and particles during their journeys. Moreover it is very simple to acquire from literature this type of measures, commonly performed in order to characterize $\gamma$-sources. And this is the best way in which we can find in literature a characterization of photon source [6] used for human body irradiation.

In the chosen application, human body represents the transport medium. This case study considers that the primary scans are multiple 2D Computed axial tomographies (CT); from these tomographies the SW builds a 3D model of the domain. The radiation source can be a real $\gamma$ beam.

The most commonly used representations are: Percentage Depth-Dose (PDD), Cross Beam Profile (CBP); we have found references about ${ }^{60} \mathrm{Co} \gamma$-decay source and tungsten bremmstrahlung X-ray sources, these last based on linearly accelerated electron beam at various peak energies.

We needed to enrich the model and the code in order to be able to use the transport simulator in a context where human body represents the medium; in this framework the designed software gives back a 3D intensity color graph where the results are fused with CT scan data: color's tonality returns info about total released energy, brightness about density of the "affected volume".

\section{Results}

In these last cards we can see the different attitude of examined radiation sources to reach deep region inside medium-high density matter (water, $\rho=1$ $\mathrm{g} / \mathrm{cm}^{3}$ ). We have considered also Crystal Undulator Laser (CUL) in addition to the abovementioned hi-energy radiation sources. CUL is a very recent innovation in hard $\mathrm{MeV}$ sources, and subject of on-going research [1].

Despite its novelty, we think it is very interesting to consider it as a term of comparison due to its unique features: above all the capability to produce a spectrum peaked in deeply penetrating photons, extremely convergent and devoid of the low-penetrating soft X-ray components (harmful, the most of times). 

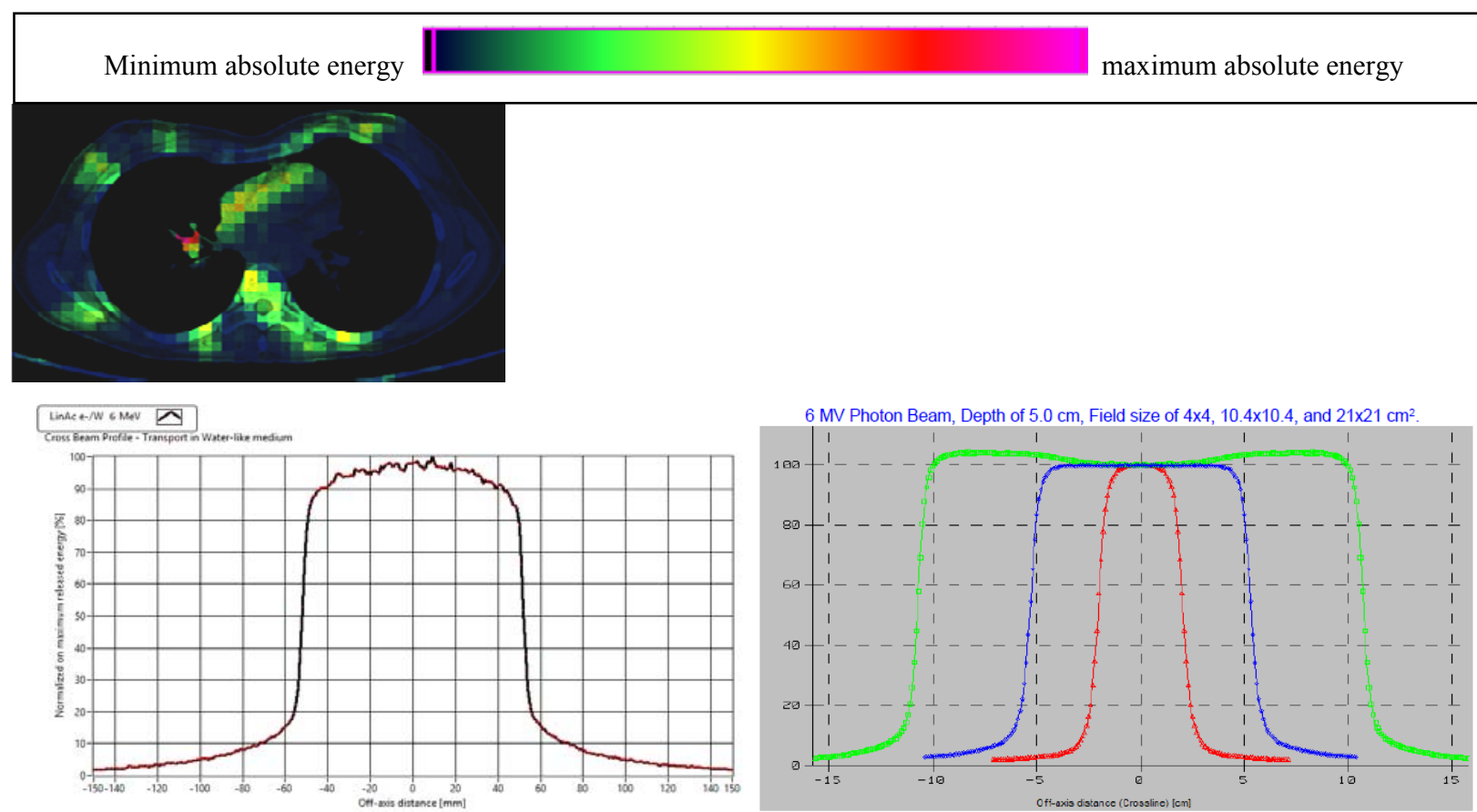

Fig. 1 CBP curves related to a source exploiting bremmstrahlung X-ray source from Linac $6 \mathrm{MeV}$ electron with a fluxing area of $104 \times 104 \mathrm{~mm}^{2}$. On the left results obtained employing our system. On the right are diagrammed data taken from “Hospital physicists association, central axis depth dose data for use in radiotherapy, Br J. Radiol 1978”.
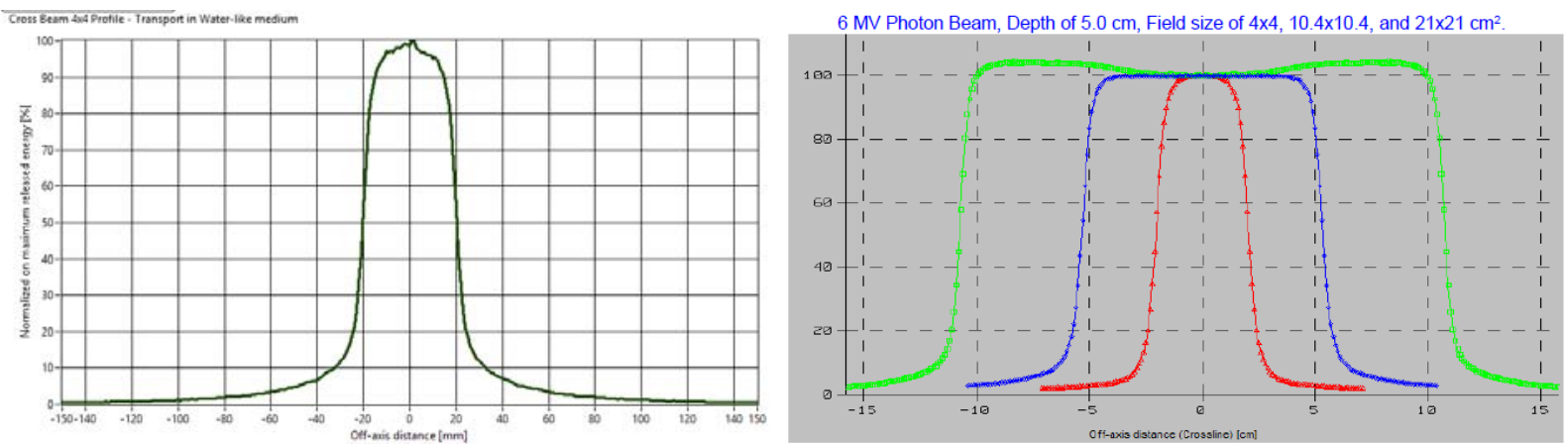

Fig. 2 CBP curves related to a source exploiting bremmstrahlung X-ray source from Linac $6 \mathrm{MeV}$ electron with a fluxing area of $40 \times 40 \mathrm{~mm}^{2}$. On the left, results obtained employing our system. On the right are diagrammed data taken from “Hospital physicists association, central axis depth dose data for use in radiotherapy, Br J. Radiol 1978".
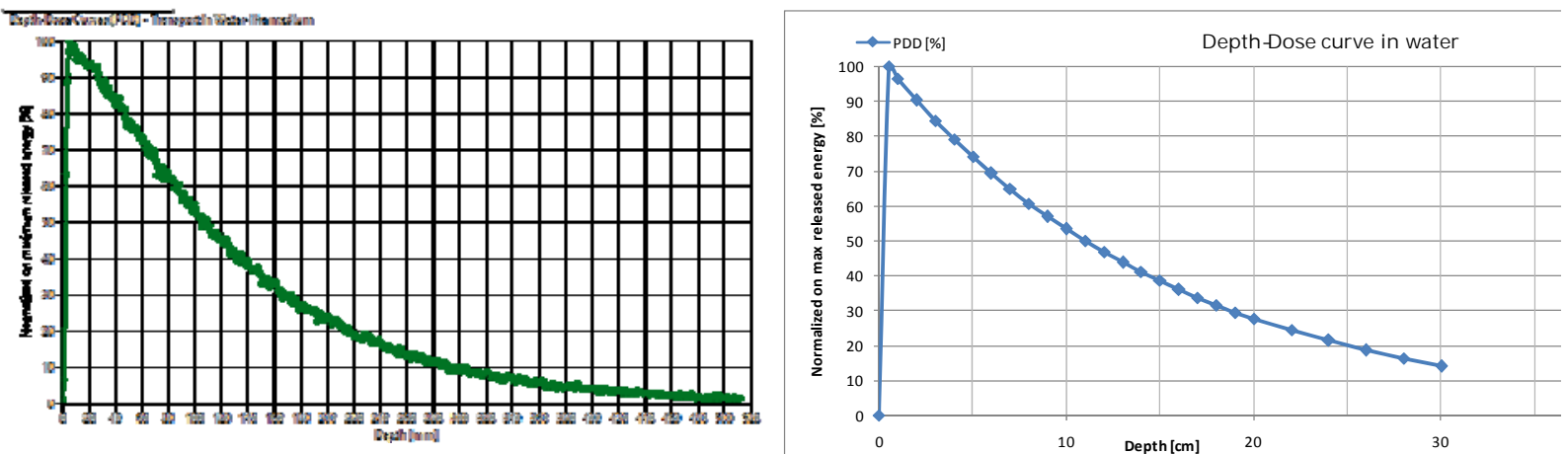

Fig. 3 PDD curves related to a source exploiting ${ }^{60} \mathrm{Co}$ decaying core with a fluxing area of $40 \times 40 \mathrm{~mm}^{2}$. On the left, results obtained employing our system. On the right are diagrammed data taken from "Hospital physicists association, central axis depth dose data for use in radiotherapy, Br J. Radiol 1978”. 

Radiation Sources and Radiation Transport inside Matter
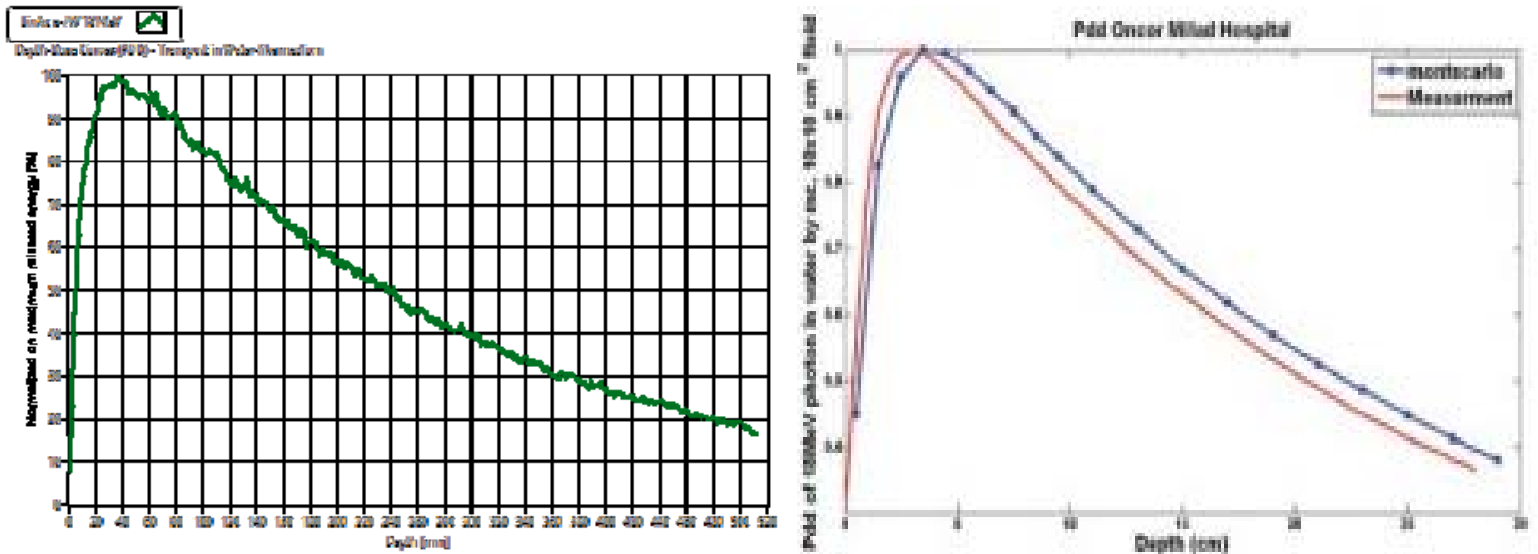

Fig. 4 PDD curves related to a source exploiting bremmstrahlung Xray source from Linac $18 \mathrm{MeV}$ electron with a fluxing area of $10 \times 10 \mathrm{~mm}^{2}$. On the left results obtained employing our system. On the right are diagrammed data taken from “Jabbari K, Anvar HS, Tavakoli MB, Amouheidari A, Monte Carlo Simulation of Siemens ONCOR Linear Accelerator with BEAMnrc and DOSXYZnrc Code, J. Med. Signals Sens (2013)”.
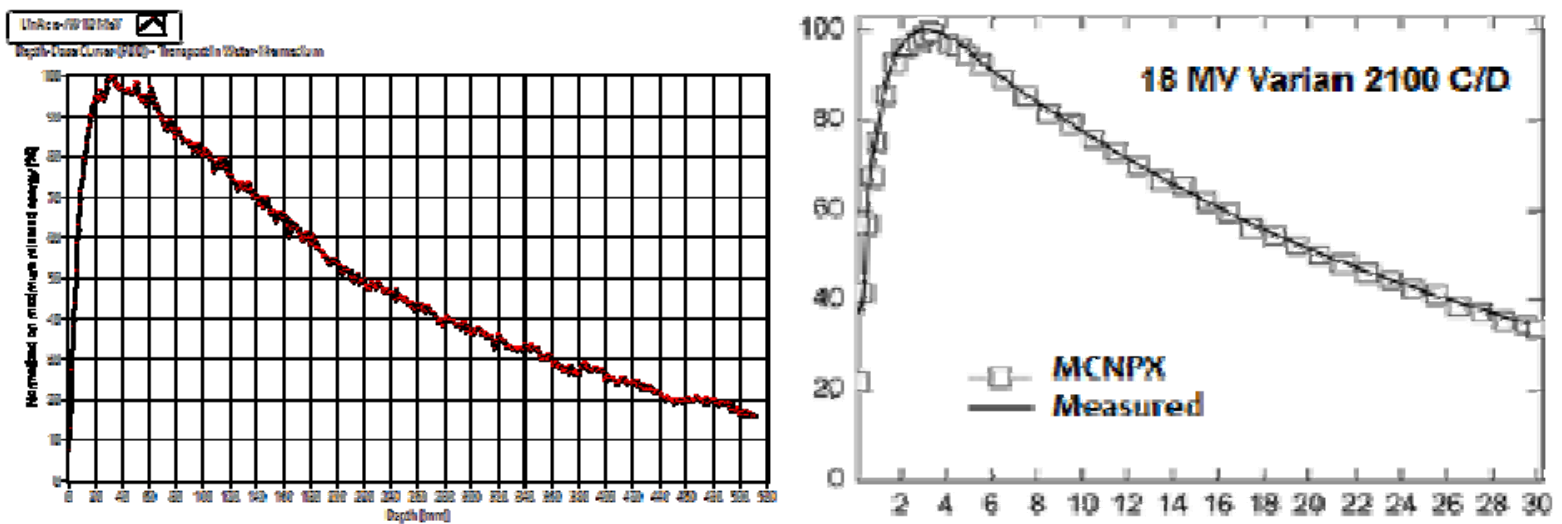

Fig. 5 PDD curves related to a source exploiting bremmstrahlung Xray source from Linac $18 \mathrm{MeV}$ electron with a fluxing area of $40 \times 40 \mathbf{m m}^{2}$. On the left results obtained employing our system. On the right are diagrammed data taken from "H. R. Vega-Carrillo, S. A. Martínez-Ovalle, J. L. Benites-Rengifo, A. M. Lallena, Photon and photoneutron spectra produced in radiotherapy LINACs, 2011".
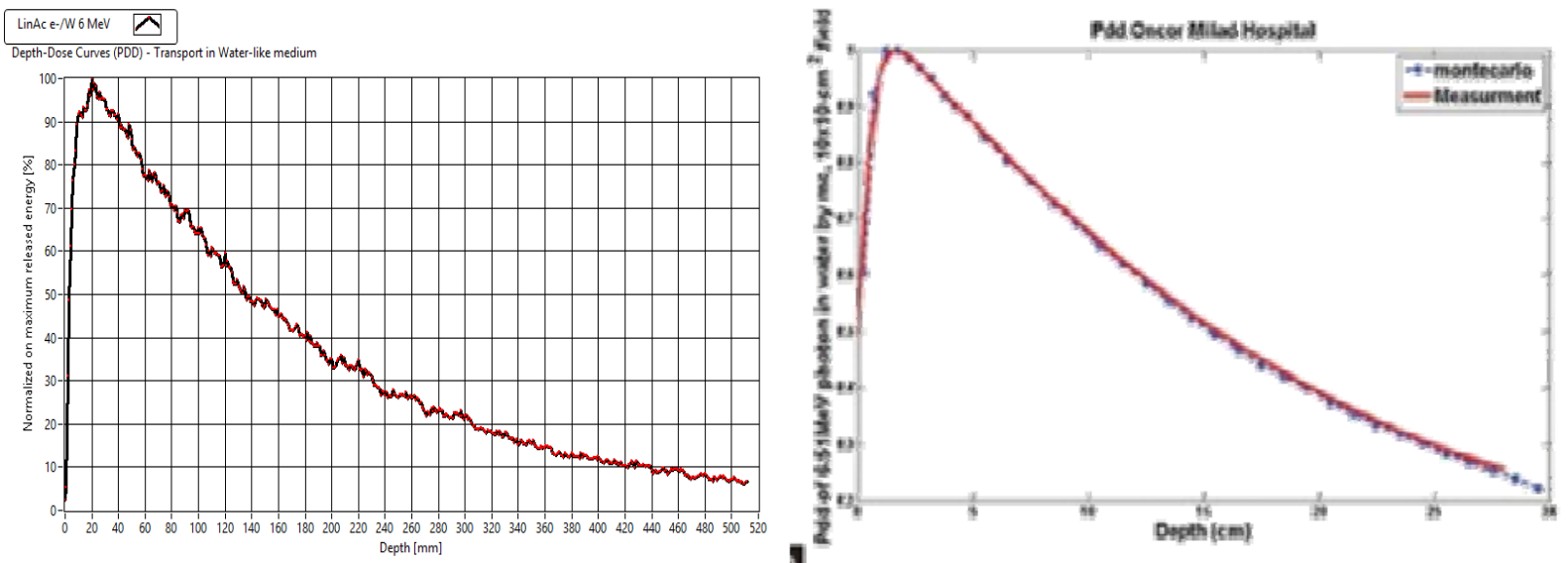

Fig. 6 PDD curves related to a source exploiting bremmstrahlung X-ray source from Linac $6 \mathrm{MeV}$ electron with a fluxing area of $40 \times 40 \mathrm{~mm}^{2}$. On the left results obtained employing our system. On the right are diagrammed data taken from “Jabbari K, Anvar HS, Tavakoli MB, Amouheidari A, Monte Carlo Simulation of Siemens ONCOR Linear Accelerator with BEAMnrc and DOSXYZnrc Code, J. Med. Signals Sens (2013)”. 


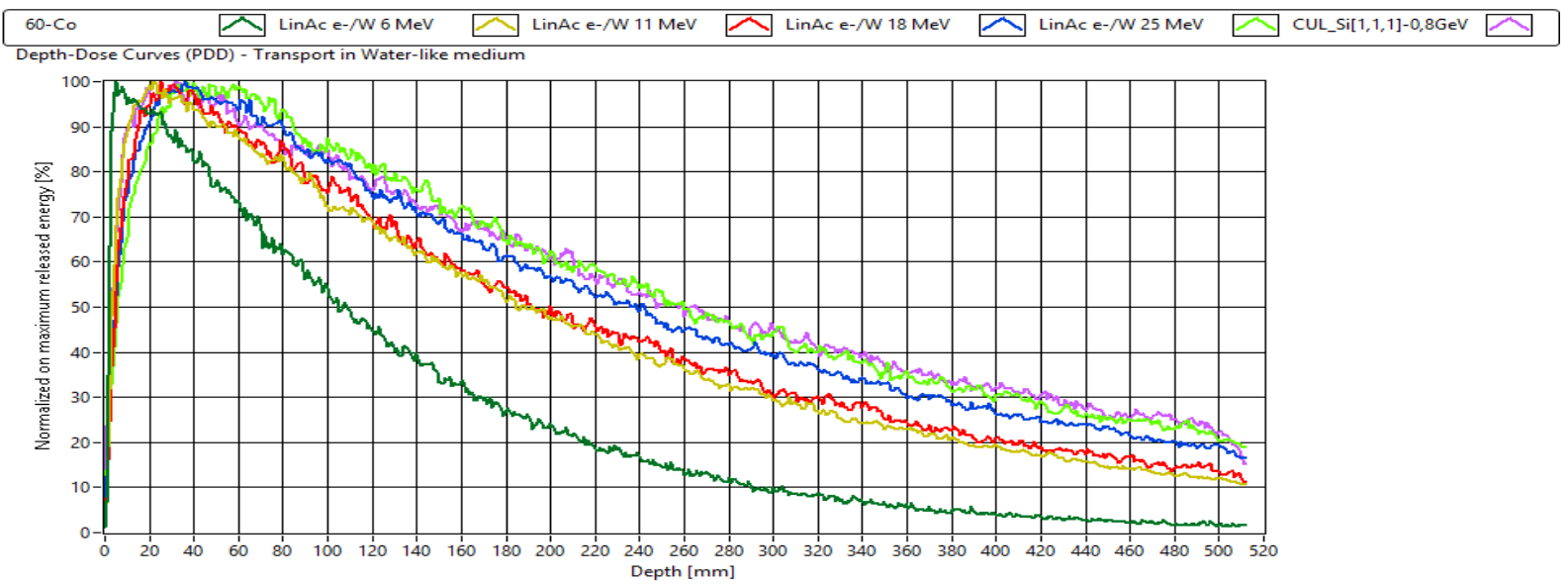

CUL uses monocrystalline lattices as source of undulator radiation when they are hit by a high energy collimated $\beta$-beam: we have extrapolated a serviceable model in terms of spectral composition and radiation diagram of output beam, exploiting available literature [1] in this young field of research.

However it should be pointed out that this type of device needs primary electron radiation beams with exceptional features, but we consider very close the time in which such a beams will be "easily" available, thanks to all the recent progress of laser drive wake-field accelerator techniques [8, 9], and also considering that exploitation of these electron beams in medical radiation is already a reality [10].

\section{Conclusions}

A new system for quantum transport has been designed with a programming environment previously not used for missions of this typology. The system is greatly reliable; we have collected many test data, subjected to tolerable level of errors.

Times required for processing are consistent with other similar application: Our calculator solves 40 particles per second on average (equipped with Intel i7 3632QW and 18\% computational power constantly exploited by the simulator).

These returned results are very useful to judge which source and which strategy of irradiation would be most useful to obtain a preferred deeper deposition of energy in the medium. But many other immediate applications in medical physics are obtainable such as MC solver for brachytherapy dose distribution problem; PET 3D image reconstruction entirely based on $\mathrm{MC}$ approach.

\section{References}

[1] Hubbell, J. H., and Seltzer, S. M. 1996. "Tables of X-Ray Mass Attenuation Coefficients and Mass Energy-Absorption Coefficients from $1 \mathrm{keV}$ to $20 \mathrm{MeV}$ for Elements $Z=1$ to 92 and 48 Additional Substances of Dosimetric Interest.” Radiation Physics Division, PML, NIST (C) 1989, 1990, 1996 Copyright by the U.S. Secretary of Commerce.

[2] Di Carlo, A., and Lugli, P. 2003. "Appunti di Optoelettronica, volume secondo: materiali semiconduttori." ISBN 88-7999-332-1, pp. 72-3, March 2003.

[3] Corvisiero, P. Handouts. Available at https://www.ge.infn.it/ corvi/doc/didattica/radioattivita/le zioni/, unpublished.

[4] Ceradini, F. 2003. "Appunti del corso di istituzioni di Fisica Nucleare e Subnucleare." unpublished.

[5] Wichmann, B. A., and Hill, I. D. 1982. "An Efficient and Portable Pseudo-Random Number Generator.” Applied Statistics 31 (2): 188-90.

[6] Frank Verhaegen and Jan Seuntjens. 2003. "Monte Carlo Modelling of External Radiotherapy Photon Beams." Phys. Med. Biol. 48: R107-64.

[7] Sushko, G. B., Korol, A. V., Greiner, W., and Solov'yov, A. V. 2013. "Sub-GeV Electron and Positron Channeling in Straight, Bent and Periodically Bent Silicon Crystals" Journal of Physics Conference Series 438 (438): 012018.

[8] Banerjee, S., Powers, N., Ramanathan, V., et al. 2012. Generation of Tunable, 100-800 MeV Quasi-monoenergetic Electron Beams from a Laser-Wakefield Accelerator in the Blowout Regime. 
Donald Umstadter Publications, 82.

[9] Wang, X., et al. 2013. "Quasi-monoenergetic Laser-Plasma Acceleration of Electrons to $2 \mathrm{GeV}$." Nat Commun. 4: 1988. Doi: 10.1038/ncomms2988.
[10] Nakajima, K., Yuan, J., Chen, L., and Sheng, Z. 2015. "Laser-Driven Very High Energy Electron/Photon Beam Radiation Therapy in Conjunction with a Robotic System.” Applied Sciences. 